\title{
Kepler detection of a new extreme planetary system orbiting the subdwarf-B pulsator KIC 10001893
}

\author{
R. Silvotti ${ }^{1}$, S. Charpinet ${ }^{2,3}$, E. Green ${ }^{4}$, G. Fontaine ${ }^{5}$, J. H. Telting ${ }^{6}$, R. H. Østensen ${ }^{7}$, \\ V. Van Grootel ${ }^{8}$, A. S. Baran ${ }^{9}$, S. Schuh ${ }^{10,11}$, and L. Fox Machado ${ }^{12}$ \\ ${ }^{1}$ INAF - Osservatorio Astrofisico di Torino, via Osservatorio 20, 10025 Pino Torinese, Italy \\ e-mail: silvotti@oato.inaf.it \\ 2 Université de Toulouse, UPS-OMP, IRAP, 31400 Toulouse, France \\ 3 CNRS, IRAP, 14 Av. E. Belin, 31400 Toulouse, France \\ ${ }^{4}$ Steward Observatory, University of Arizona, 933 North Cherry Avenue, Tucson, AZ 85721, USA \\ 5 Département de Physique, Université de Montréal, CP 6128, Succ. Centre-Ville, Montréal, Québec H3C 3J7, Canada \\ ${ }^{6}$ Nordic Optical Telescope, Rambla José Ana Fernández Pérez 7, 38711 Breña Baja, Spain \\ 7 Instituut voor Sterrenkunde, KU Leuven, Celestijnenlaan 200D, 3001 Leuven, Belgium \\ 8 Institut d'Astrophysique et de Géophysique, Université de Liège, 17 allée du 6 Août, 4000 Liège, Belgium \\ 9 Mt Suhora Observatory, Cracow Pedagogical University, ul. Podchorazych 2, 30-084 Krakow, Poland \\ 10 Institut für Astrophysik, Georg-August-Universität Göttingen, Friedrich-Hund-Platz 1, 37077 Göttingen, Germany \\ 11 Max Planck Institute for Solar System Research, Max-Planck-Strasse 2, 37191 Katlenburg-Lindau, Germany \\ 12 Observatorio Astronómico Nacional, Universidad Nacional Autónoma de México, BC, 22860, Ensenada, Mexico
}

Received 1 July 2014 / Accepted 5 September 2014

\begin{abstract}
KIC 10001893 is one out of 19 subdwarf-B (sdB) pulsators observed by the Kepler spacecraft in its primary mission. In addition to tens of pulsation frequencies in the $g$-mode domain, its Fourier spectrum shows three weak peaks at very low frequencies, which is too low to be explained in terms of $g$ modes. The most convincing explanation is that we are seeing the orbital modulation of three Earth-size planets (or planetary remnants) in very tight orbits, which are illuminated by the strong stellar radiation. The orbital periods are $P_{1}=5.273, P_{2}=7.807$, and $P_{3}=19.48 \mathrm{~h}$, and the period ratios $P_{2} / P_{1}=1.481$ and $P_{3} / P_{2}=2.495$ are very close to the $3: 2$ and 5:2 resonances, respectively. One of the main pulsation modes of the star at $210.68 \mu \mathrm{Hz}$ corresponds to the third harmonic of the orbital frequency of the inner planet, suggesting that we see, for the first time in an sdB star, $g$-mode pulsations tidally excited by a planetary companion. The extreme planetary system that emerges from the Kepler data is very similar to the recent discovery of two Earth-size planets orbiting the sdB pulsator KIC 05807616 (Charpinet et al. 2011a).
\end{abstract}

Key words. planetary systems - stars: horizontal-branch - stars: oscillations - asteroseismology - techniques: photometric techniques: radial velocities

\section{Introduction}

What happens to the planets, in particular to the inner planets, at the end of stellar evolution is largely unknown. After the main sequence, dramatic changes in the planetary orbits and even the complete evaporation of the inner planets can occur, following the red giant branch (RGB) and asymptotic giant branch (AGB) expansion of the host star (Villaver \& Livio 2007, 2009). During the RGB or AGB expansion, the opposite effects of stellar mass loss and tidal interactions may determine a gap in the final distribution of orbital distances and periods (Nordhaus \& Spiegel 2013). The first step in studying the effects of the RGB expansion is to search for planetary systems on the horizontal branch (HB). The star studied in this article is a subdwarf B (sdB), located on the extreme horizontal branch (EHB).

The sdB stars are a homogeneous class of very hot objects with a mass distribution peaked near $0.47 M_{\odot}$ (Fontaine et al. 2012) and with a very thin hydrogen envelope (see the review by Heber 2009 for more details on hot subdwarfs). Almost all the envelope was lost near the tip of the RGB. Such strong envelope ejection is explained well in terms of close binary evolution for half of the sdBs that have a close stellar companion, generally an M dwarf or a white dwarf (Han et al. 2002, 2003). But it is more problematic for the other half of apparently single sdB stars. The presence of close and massive planets or brown dwarfs (BDs) is a possible explanation (Soker 1998; Nelemans \& Tauris 1998), that seems corroborated by recent calculations (Han et al. 2012).

The first detections of substellar companions to sdB stars started seven years ago, and now we know about thirteen sdB stars with planet/BD candidates belonging to three different groups that are quite distinct in terms of orbital distance and planetary mass. In order of decreasing orbital distance we find: i) eight planet/BD candidates in wide orbits (orbital periods between 3.2 and $\sim 16$ yrs), with masses between $\sim 2$ and $\sim 40 M_{\text {Jup }}$ (Silvotti et al. 2007; Lee et al. 2009; Beuermann et al. 2012a,b; Qian et al. 2009, 2012; Lutz 2011; Schuh et al. 2014); ii) two Earth-mass planet candidates around the sdB pulsator KIC 05807616, with orbital periods of 5.8 and $8.2 \mathrm{~h}$ (Charpinet et al. 2011a); iii) at least two BD candidates with short orbital periods of a few hours (Geier et al. 2011; Schaffenroth et al. 2014; see also Geier et al. 2012). These three 
groups correspond to three different detection methods - timing, illumination effects, and radial velocities (RVs), respectively that sample three different regions of the $a-m_{\mathrm{p}}$ (semi-major axis vs. planetary mass) plane (see Fig. 1 of Silvotti et al. 2014).

The planet candidates presented in this paper belong to the second group and closely resemble the planets of KIC 05807616. These two planetary systems show very similar characteristics (both with two or three Earth-size planets very close to the parent star and very hot), suggesting that these extreme planetary systems around sdB stars might not be so rare and implying that planets could indeed play a role during the envelope ejection that is needed to form an $\mathrm{sdB}$ star.

The subject of this article has implications in different overlapping fields: sdB evolution, common envelope (CE) ejection mechanisms, survival of a planet to a CE phase, and planetary system evolution in general.

\section{Kepler data and ephemeris}

With a Kepler magnitude of 15.85 , KIC 10001893 is one out of 19 subdwarf B (sdB) pulsators observed by the Kepler spacecraft in its primary mission (Østensen et al. 2010, 2011, 2012; Kawaler et al. 2010a,b; Reed et al. 2010, 2011, 2012; Van Grootel et al. 2010; Baran et al. 2011, 2012; Charpinet et al. 2011b). The spectroscopic atmospheric parameters of KIC 10001893, $T_{\text {eff }}=26700 \pm 300 \mathrm{~K}, \log g=5.30 \pm 0.04$, and $\log \left(N_{\mathrm{He}} / N_{\mathrm{H}}\right)=-2.09 \pm 0.1$ are very compatible with the $g$-mode instability strip (Østensen et al. 2011). The star was observed intensively with the Kepler spacecraft (Borucki et al. 2010) in the framework of the Kepler Asteroseismic Science Consortium (KASC, Gilliland et al. 2010), mostly in short cadence mode (SC, 58.85 s sampling), for 36 monthly runs (period Q3.2, where "Q" stands for quarter, and continuously from Q6 to Q17.2, when the spacecraft lost its second of four gyroscope-like reaction wheels). Moreover, it was observed in long-cadence mode (LC, 29.4 min sampling) for five more months (Q3.1, Q3.3, and Q5). In our analysis we used all the available data, which means $993.8 \mathrm{~d}$ of SC data and $147.9 \mathrm{~d}$ of LC data. The data were downloaded from the Kepler Asteroseismic Science Operations Center (KASOC) website ${ }^{1}$.

From a preliminary analysis using the photometric data produced by the Kepler pipeline, the Fourier transform shows a rich spectrum with more than one hundred peaks, mostly concentrated between 76 and $441 \mu \mathrm{Hz}$. At the low-frequency end of the amplitude spectrum, our attention was caught by a few peaks at very low frequency below $60 \mu \mathrm{Hz}$, that exceed the mean noise level by about four times (upper panel of Fig. 1). These frequencies are too low to be produced by $g$-mode oscillations considering that the $\ell=1(\ell=2)$ atmospheric cut-off for the gravity modes is near $60(105) \mu \mathrm{Hz}$ for a star like KIC 10001893 (Hansen et al. 1985; Charpinet et al. 2011a). At lower frequencies, the reflective boundary condition at the surface is no longer valid.

To verify that these low-frequency peaks could be produced by a nearby star located at approximately 8.5 arcsec northeast of KIC 10001893, we decided to use the pixel data that are available at the KASOC website and extract the photometry again for both the target and the close star, thereby optimizing the pixel combinations. The photometric contamination from nearby stars is a serious problem given the Kepler pixel size of about 3.98 arcsec. For the close star we used only one pixel, the one

http://kasoc.phys.au.dk/kasoc/

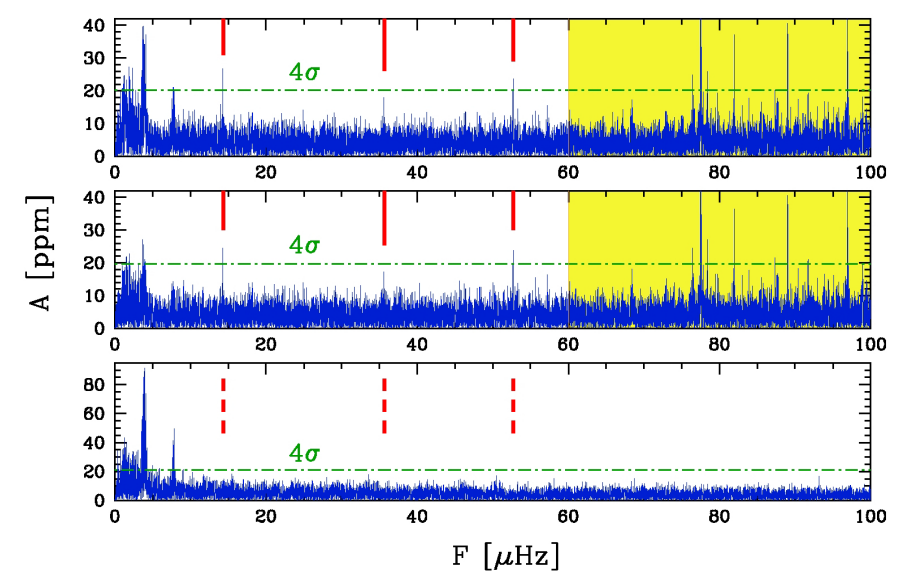

Fig. 1. Fourier transform of KIC 10001893 at low frequency. The three panels show the amplitude spectrum using the standard photometry produced by the Kepler pipeline (top), the pixel photometry obtained optimizing the pixels on the target star (center), and the pixel photometry using only 1 pixel, centered on the nearby star (bottom). The plot demonstrates that the frequency at about $4 \mu \mathrm{Hz}$ and its first harmonic are caused by the nearby star, while the three other peaks (marked with tick vertical lines, red in the electronic version) are intrinsic to KIC 10001893. The shaded area at frequency higher than $60 \mu \mathrm{Hz}$ (yellow in the electronic version) represents the region of the $g$-mode oscillations.

corresponding to the PSF maximum of this star, so that the contribution from KIC 10001893 was reduced to almost zero. For the target we opted for a solution that could, at the same time, minimize the photons from the nearby star and also maximize the signal-to-noise ratio $(\mathrm{S} / \mathrm{N})$ : after different attempts, we decided to use, in each quarter, the pixel combination that was giving the maximum amplitude to the pulsation frequencies in the Fourier transform. In Fig. 1 the amplitude spectrum of the two stars is shown.

We see very well that the peak at $4 \mu \mathrm{Hz}$ and its harmonic near $8 \mu \mathrm{Hz}$ are produced by the nearby star (lower panel of Fig. 1). Less clear is the origin of some power near $2 \mu \mathrm{Hz}$, but at $\sim 2 \mu \mathrm{Hz}$ instrumental drifts may already have some importance. On the other hand, it is clear from Fig. 1 that the three frequencies $f_{1}=52.68 \mu \mathrm{Hz}, f_{2}=35.58 \mu \mathrm{Hz}$, and $f_{3}=14.26 \mu \mathrm{Hz}$, corresponding to the periods $P_{1}=5.27 \mathrm{~h}, P_{2}=7.81 \mathrm{~h}$, and $P_{3}=19.48 \mathrm{~h}$, are intrinsic to the sdB star. This conclusion is also confirmed from independent data processing using a model of the pixel response function determined from a combination of the Kepler optical point-spread function and various systematics of the spacecraft, and this helps to avoid contamination from the neighboring stars. When using all the available data, the amplitudes of the three orbital periods have $\mathrm{S} / \mathrm{Ns}$ of $4.8,3.5$, and 5.0 (Fig. 1). At different amplitude levels, the three peaks are also visible when we consider shorter subsets containing only one third of the data (Fig. 2). In the first subset, the peak at $35.58 \mu \mathrm{Hz}$, which was the faintest one in Fig. 1, reaches a $\mathrm{S} / \mathrm{N}$ of 5.1 (upper panel of Fig. 2). Most important, the phases of the three peaks are coherent in each independent subset. The phase-folded light curves are shown in Fig. 3, and from the upper panel we note that $P_{1}$ is very close to four times one of the main pulsation periods of the star.

We have already seen that the low-frequency variations detected cannot be explained in terms of $g$ modes. We add that they do not even correspond to linear combinations of the 


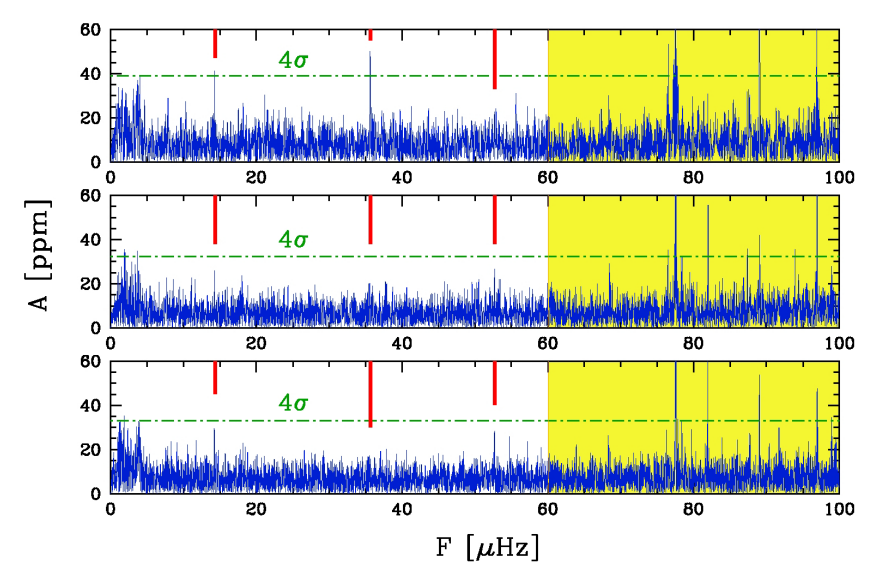

Fig. 2. Fourier transform of KIC 10001893 using three sets of independent pixel photometry data with a duration of about 13-14 months each. From top to bottom: Q3+Q5+Q6+Q7+Q8.1, Q8.2+Q8.3+Q9+Q10+ $\mathrm{Q} 11+\mathrm{Q} 12.1+\mathrm{Q} 12.2, \mathrm{Q} 12.3+\mathrm{Q} 13+\mathrm{Q} 14+\mathrm{Q} 15+\mathrm{Q} 16+\mathrm{Q} 17.1+\mathrm{Q} 17.2$. The apparent changes in amplitude of the low-frequency peaks are mostly consistent with statistical noise.

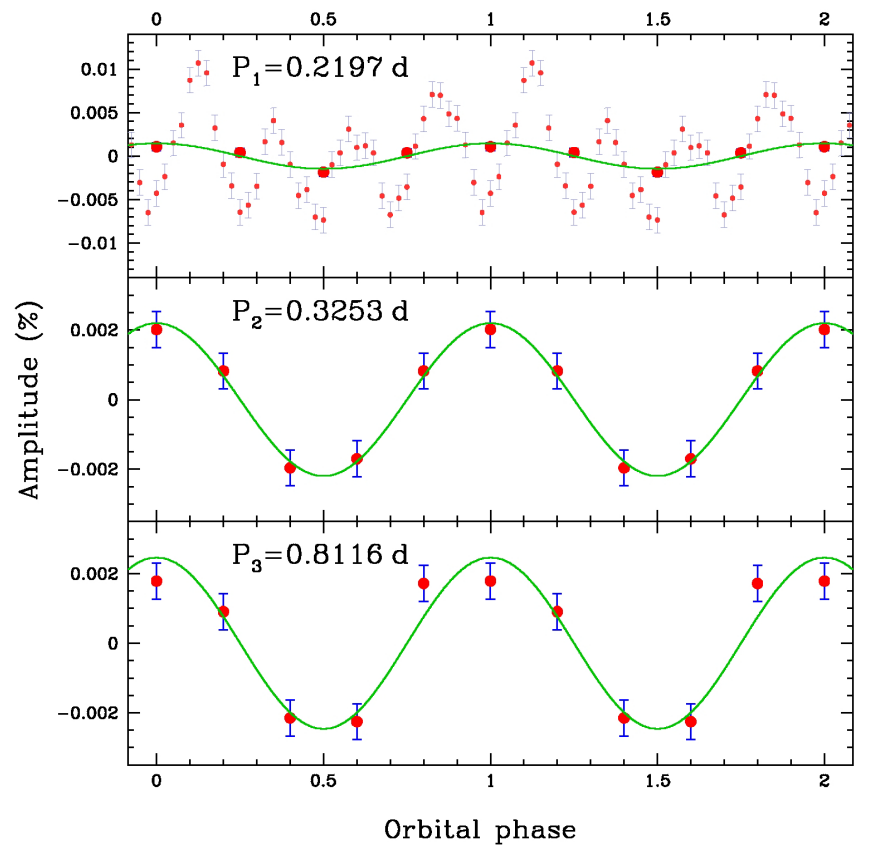

Fig. 3. Phase-folded light curves for the three planet candidates and our best fits with a sine wave. Each point is the mean flux in that phase bin. The upper panel shows that the inner planet has a period very close to 4 times one of the main pulsation periods of the star. The error bars are obtained from the original flux uncertainties given by the Kepler pipeline: $\sigma=\frac{1}{n}\left(\sum_{i=1}^{n} \sigma_{i}^{2}\right)^{1 / 2}$.

pulsation frequencies ${ }^{2}$. Other explanations like surface spots are very unlikely. Spots have never been observed in sdB stars, which have stable radiative envelopes (no convection) and are

\footnotetext{
2 The maximum coincidence is for $f_{1}=52.681 \mu \mathrm{Hz}$, so relatively close to the difference between two low-amplitude peaks: 204.670$152.018=52.652 \mu \mathrm{Hz}$ (the formal resolution being $0.013 \mu \mathrm{Hz}$ ). However, it would be very surprising that these faint peaks (37 and $67 \mathrm{ppm}$, respectively) give rise to linear combinations, while no combinations peaks are seen for much stronger pulsation frequencies. On the other hand, $f_{2}=35.582 \mu \mathrm{Hz}$ is not very far from 359.680 $323.980=35.700 \mu \mathrm{Hz}$, two high-amplitude peaks (520 and $823 \mathrm{ppm}$, respectively), but here the difference in frequency is nine times the frequency resolution.
}

non-magnetic. Indeed we do not see any signature of a magnetic field in our optical spectra or in the pulsation spectrum. Moreover, sdB stars have relatively long rotational periods, $P_{\text {ROT }}>1$ day from spectroscopy (Geier \& Heber 2012), while values up to $\sim 40$ days are obtained from pulsation rotational splitting (e.g., Baran et al. 2012). This rules out all (known) alternatives other than orbitally driven modulations. In conclusion, the most convincing explanation for the three low-frequency variations observed in the light curve of KIC 10001893 is the presence of three low-mass bodies orbiting the star in tight orbits.

A strong argument in favor of this interpretation is given by the period ratios $P_{2} / P_{1}=1.480$ and $P_{3} / P_{2}=2.495$, which are very close to the $3: 2$ and 5:2 resonances. Another interesting aspect is that one of the main pulsation modes of the star, the one at $210.68 \mu \mathrm{Hz}$, corresponds to the third harmonic of the orbital frequency of the inner planet $(52.68 \times 4=210.72 \mu \mathrm{Hz})$, suggesting a tidal resonance (upper panel of Fig. 3$)^{3}$. Although tidally excited pulsations have already been observed in other stars (e.g., Hambleton et al. 2013), this is the first time that we have a clear indication of this phenomenon in an $\mathrm{sdB}$ star.

Fitting the Kepler data with sinusoidal waves, it is possible to compute the ephemeris of the three planet candidates:

$$
\begin{aligned}
& \mathrm{BJD}_{1}^{\mathrm{TDB}}=2455093.4293(53)+0.2197(34) E \\
& \mathrm{BJD}_{2}^{\mathrm{TDB}}=2455093.325(44)+0.3253(59) E \\
& \mathrm{BJD}_{3}^{\mathrm{TDB}}=2455093.506(41)+0.8116(62) E .
\end{aligned}
$$

These equations give the times of the maximum flux, when each planet is at the maximum distance from our solar system (phase 0 in Fig. 3). The reference epochs correspond to the first time at phase 0 of the Kepler data. The relatively large errors in Eqs. (1)-(3), obtained from Monte-Carlo simulations, are due to the extremely faint signals. A word of caution is needed about the phases, which are not very reliable. From various tests, we have seen that the phases can change significantly when we change the number of pulsation frequencies included in the fit. The phases reported here were computed using 60 pulsation frequencies with amplitudes greater than $20 \mathrm{ppm}$.

Unfortunately, despite the very high quality of the Kepler data, it is not possible to confirm the presence of the three planets by an independent method using the same data. All the methods that make use of the Römer delay on pulsation timing - O-C method (Silvotti et al. 2007), light curve fitting (Telting et al. 2012), and orbital aliases in the Fourier domain (Telting et al. 2012; Shibahashi \& Kurtz 2012) - require larger orbits. With these short orbital periods, the maximum light-travel time shift is only $\left(1.8 \times 10^{-5} M_{\mathrm{PE}} \sin i\right)$, $\left(2.3 \times 10^{-5} M_{\mathrm{PE}} \sin i\right)$, and $\left(4.2 \times 10^{-5} M_{\mathrm{PE}} \sin i\right)$ seconds (with the planet mass $M_{\mathrm{PE}}$ in Earth masses, where $i$ is the inclination of the system with respect to the line of sight). And the amplitude of the first orbital sidelobes at $\pm v_{\text {orb }}$ from the main pulsation frequency at $274.302 \mu \mathrm{Hz}$ (which has an amplitude of $0.1 \%$ ) would be $\left(3.0 \times 10^{-5} M_{\mathrm{PE}} \sin i\right),\left(4.0 \times 10^{-5} M_{\mathrm{PE}} \sin i\right)$, and $\left(7.3 \times 10^{-5} M_{\mathrm{PE}} \sin i\right) \mathrm{ppm}$. These numbers are well below the detection limit of Kepler for any realistic planetary mass. 3 The period ratios close to resonances and the relation between $P_{1}$ and
one of the main pulsation periods of the star allow to exclude also that
the photometric variations that we see are produced by any instrumental effects. 


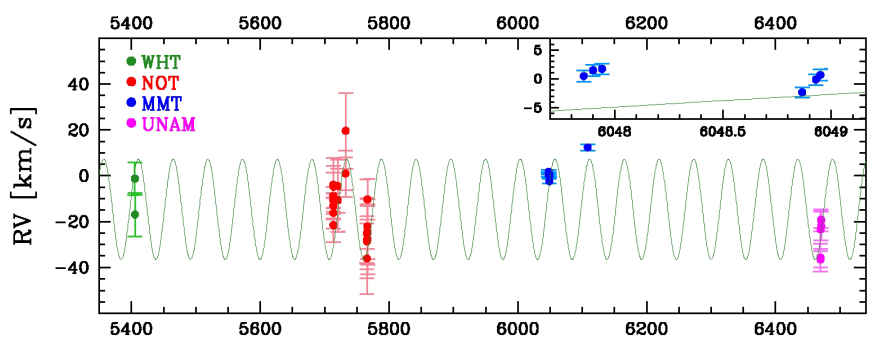

Fig. 4. Radial velocities of KIC 10001893. The inset shows in detail the MMT measurements of April 30 and May 1, 2012. A tentative fit with a period of about 54 days is shown.

\section{Spectroscopy and radial velocities}

KIC 10001893 was observed in 2010, 2011, 2012, and 2013 using the William Herschel, the Nordic Optical, the Multi Mirror, and the $2.1 \mathrm{~m}$ San Pedro Martir Telescopes (WHT, NOT, MMT, and UNAM) to measure possible radial velocity (RV) variations (Fig. 4).

The six best RV measurements from the MMT on April 30 and May 1, 2012 are consistent with a constant radial velocity of $0.3 \pm 1.6 \mathrm{~km} \mathrm{~s}^{-1}$. However, a single MMT spectrum taken two months later on June 30 indicates a RV of $12.3 \pm 1.3 \mathrm{~km} \mathrm{~s}^{-1}$. We do not have any reason to think that this last measurement has any problem, because spectra taken on the same night on another target, Feige 48 , agree to better than $1 \mathrm{~km} \mathrm{~s}^{-1}$ with the binary orbit of Feige 48. Unfortunately, we did not have the possibility to observe KIC 10001893 again with MMT in 2012, 2013, or 2014. Our conclusion is that with the RV data that we have we can exclude any RV amplitude larger than about $25 \mathrm{~km} \mathrm{~s}^{-1}$. Considering only the MMT data (last spectrum included), the amplitude must be at least $6 \mathrm{~km} \mathrm{~s}^{-1}$. If we associate an RV variation of this amplitude with the longer orbital period that we found in the Kepler data (19.5 h), the minimum mass of the companion (at $i=90^{\circ}$ ) would be about 17 Jupiter masses, which is enough to make the system dynamically unstable. Thus, if the $\mathrm{RV}$ variation is real, we should instead think of a more massive faint (stellar) companion in a wide orbit ${ }^{4}$. A tentative fit of the $\mathrm{RV}$ data with a period of about 54 days is shown in Fig. 4.

With the many spectra that we got on KIC 10001893 (including some spectra from the Bok telescope), it was possible to improve the atmospheric parameters of the star. The WHT and NOT spectra were fit using LTE models with solar abundances (Heber et al. 2000), while NLTE models were used to fit the Bok and MMT spectra. Our best estimate is $T_{\text {eff }}=27500 \pm 500$, $\log g=5.35 \pm 0.05$ and $\log \left(N_{\mathrm{He}} / N_{\mathrm{H}}\right)=-2.95 \pm 0.04$.

\section{An extreme planetary system}

In this section we explore the properties of the three low-mass bodies according with the variations observed in the light curve. As discussed by Charpinet et al. (2011a), the light modulation that we see can be caused by the reflected light from the illuminated side of each low-mass companion. The intensity of this effect depends on the Bond albedo $\alpha_{\mathrm{B}}$. Another effect, which can be even more important for very hot stars and very close planets, is the thermal emission from each planet, which is modulated by the temperature difference between the heated day side and the cooler night-side hemisphere, assuming that the rotation of each

\footnotetext{
4 The absence of Doppler beaming and ellipsoidal deformation of the sdB star also allow excluding that the photometric variations that we see are caused by a white dwarf companion in a close orbit.
}

planet is tidally synchronized to its orbital motion (a likely situation given the short orbital periods). Under the assumption of radiative equilibrium and black-body re-emission, we introduce a second parameter $\beta$, defined as the ratio between the two temperatures. The equilibrium temperatures on the two hemispheres are given by

$T_{\text {day }}=\left[\frac{1-\alpha_{\mathrm{B}}}{2\left(1+\beta^{4}\right)}\right]^{1 / 4}\left(R_{\star} / a\right)^{1 / 2} T_{\text {eff }}$

$T_{\text {night }}=\beta T_{\text {day }}$

where $a=\left[G M_{\star} /\left(4 \pi^{2}\right)\right]^{1 / 3} P_{\text {orb }}^{2 / 3}$ is the orbital separation (assuming $\left.M_{\mathrm{P}} \ll M_{\star}\right), M_{\mathrm{P}}$ is the planet mass, $R_{\star}=\left(G M_{\star} / g\right)^{1 / 2}$ and $T_{\text {eff }}$ are the radius and the spectroscopic effective temperature of the parent star. The surface gravity $g$ is obtained from spectroscopy, and we assumed a canonical stellar mass $M_{\star}=$ $0.47 M_{\odot}$.

Then we computed the planetary radii and masses as a function of the parameters mentioned above and of the inclination, using the following equations (see Charpinet et al. 2011a, Supplementary Information, for more details):

$$
\begin{aligned}
& R_{\mathrm{P}}=\left(\frac{A}{\sin i}\right)^{1 / 2}\left[\frac{\alpha_{\mathrm{B}}}{8 a^{2}}+\frac{1}{2 R_{\star}^{2}} \frac{F\left(T_{\text {day }}\right)-F\left(T_{\text {night }}\right)}{F\left(T_{\text {eff }}\right)}\right]^{-1 / 2} \\
& F(T)=\int B_{\lambda}(T) \epsilon_{\lambda}^{K} \mathrm{~d} \lambda \\
& M_{\mathrm{P}}=\frac{4}{3} \pi R_{\mathrm{P}}^{3} \bar{\rho}
\end{aligned}
$$

in which $A$ is the measured (semi-)amplitude of the photometric modulation, $F(T)$ is the black body radiative flux within the Kepler response function $\epsilon_{\lambda}^{K}, B_{\lambda}$ is the Planck distribution, and $\bar{\rho}$ is the mean planet density.

Finally we computed the RV amplitude $K$ of the parent star:

$$
K=\left(\frac{2 \pi G}{P}\right)^{1 / 3} \frac{M_{\mathrm{P}} \sin i}{\left(M_{\star}+M_{\mathrm{P}}\right)^{2 / 3}} .
$$

The results of our analysis are summarized in Fig. 5. We see that for any reasonable value of $\alpha_{\mathrm{B}}$ and $\beta$ (and for any inclination $\gtrsim 1$ degree), radii and masses are in the planetary range. Only with a very high albedo and a very small temperature difference between the two hemispheres (isothermal surface) can the radii be larger than the Jupiter radius if the inclination is very low. But these conditions, similar to Venus, would require a dense atmosphere, which is very difficult to imagine in an extremely hot planet in a close orbit that has presumably entered the star envelope in the past. Even in the case of secondgeneration planets, formed after the RGB phase, it is unlikely that a dense atmosphere can be kept in such extreme conditions. Assuming $\alpha_{\mathrm{B}}=0.1$ and $\beta=0.2$, the radii of the planets are smaller than Neptune's radius for almost any inclination while, for $i>14^{\circ}\left(16^{\circ}\right)$, planet 1 (2) has a radius smaller than the Earth.

\section{Stability of the orbits vs inclination of the system}

From preliminary stability computations, assuming circular coplanar orbits, and assuming that the three planets have same mean density, we see that the stability of the orbits becomes critical in relatively short times $\left(<10^{6}\right.$ yrs $)$ when $\bar{\rho}(\sin i)^{-3 / 2} \gtrsim$ $1000 \mathrm{~g} / \mathrm{cm}^{3}$. With mean densities of $1.3,5.5$, and $13.75 \mathrm{~g} / \mathrm{cm}^{3}$, 

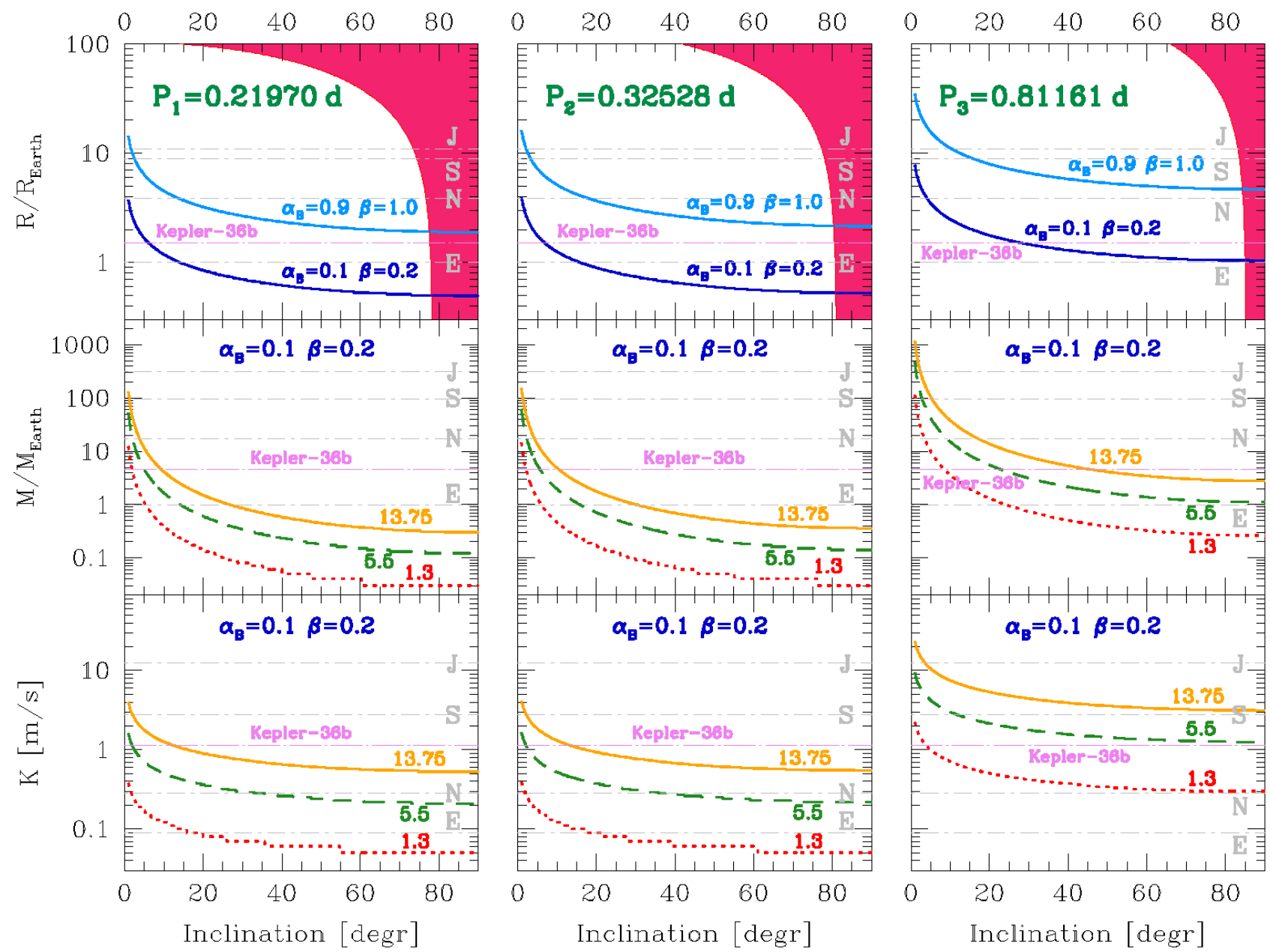

Fig. 5. Radii and masses of the three planets and host-star-projected RV amplitudes as functions of the inclination. We assume a Bond albedo $\alpha_{\mathrm{B}}=0.10$ and an average temperature contrast between night and day sides $\beta=0.2$; that is, approximately the values observed on Mercury. Only in the upper panels do we also show the opposite (less likely) extreme case with $\alpha_{\mathrm{B}}=0.90$ and $\beta=1.0$ (isothermal surface). The shaded regions (red in the electronic version) in the upper panels represent excluded domains due to the absence of eclipses. Radii, masses, and stellar RV amplitudes for Jupiter, Saturn, Neptune, the Earth, and Kepler-36b, which has an estimated mean density of $7.5 \mathrm{~g} / \mathrm{cm}^{3}$ (Carter et al. 2012), are indicated for comparison. The central and lower panels show three different curves with different mean densities: 1.3 (Jupiter), 5.5 (Earth), and $13.75 \mathrm{~g} / \mathrm{cm}^{3}$ (2.5× Earth, corresponding to the mean density of a 100\% iron planet with Earth radius, Valencia et al. 2010, Fig. 4). If KIC 10001893 b,c,d are the remnants of one or more massive planets, they could have a high density corresponding to the iron-rich cores of their Jovian progenitors.

the system is dynamically stable only if the inclination is greater than about $0.7^{\circ}, 1.8^{\circ}$, and $3.3^{\circ}$, respectively. This constraint may help to understand a peculiar characteristic of the amplitude spectrum of this star, in which we do not see any rotational splitting of the pulsation frequencies (unlike all the other sdB pulsators observed by Kepler). The absence of multiplets may be due to a very low inclination (but higher than $0.7^{\circ}$ to $3.3^{\circ}$ ) and/or to an extremely long rotation period.

\section{Discussion}

The planetary system described in this paper is a new candidate in the short list of systems with substellar companions in close orbits around evolved stars. Such systems are crucial for studying the survivability of planets during the post-MS evolution of the host star, a question that has attracted some interest over the past few years (Villaver \& Livio 2007, 2009; Nordhaus et al. 2010; Passy et al. 2012; Spiegel 2012; Mustill \& Villaver 2012; Nordhaus \& Spiegel 2013; Villaver et al. 2014). As in the case of KIC 05807616, it is likely that the three planets of KIC 10001893 have lost a significant fraction of their mass during the CE phase. Following Passy et al. (2012), their initial mass, before the plunge-in, was most likely a few Jupiter masses $\left(M_{\mathrm{J}}\right)$. Much lower values should be excluded since they would cause the drag energy to be higher than the binding energy and the planet would be destroyed. On the other hand, much higher values should also be excluded since, already at $10 M_{\mathrm{J}}$, a companion would not be significantly affected by the CE phase.

Whether a few Jupiter masses are sufficient to remove the entire envelope of the host star is not clear. Following Nordhaus \& Spiegel (2013, Fig. 2), with orbital periods of 5, 8, and $19 \mathrm{~h}$, the minimum mass to eject the CE is about 13,20 , and $35 M_{\mathrm{J}}$, respectively, assuming that all the orbital energy is used to unbind the $\mathrm{CE}\left(\alpha_{\mathrm{CE}}=1\right)$. Following Han et al. (2012), when we approach the peak distribution of sdB masses at $\sim 0.47 M_{\odot}$ (Fontaine et al. 2012), a low-mass substellar companion may be sufficient to eject the common envelope, even when only the orbital energy released during the spiral-in is considered. Adding an extra term due to thermal energy, the minimum companion mass to eject the $\mathrm{CE}$ is reduced further. An alternative scenario has been proposed by Bear \& Soker (2012) for KIC 05807616: a single massive planet $\left(M \gtrsim 5 M_{\mathrm{J}}\right)$, after having removed most of the stellar envelope, reaches the tidal-destruction radius at about $1 R_{\odot}$ from the stellar core. Here the planet's gaseous 
envelope is removed, and the metallic core is disrupted in two or more Earth-size fragments that survive within the gaseous disk and migrate to resonant orbits $(3: 2$ resonance in the case of KIC 05807616; 5:2 and 3:2 for KIC 10001893). According to the same authors, the survival of the planets to the high evaporation rate due to the stellar UV radiation could be guaranteed by a planetary magnetic field ten times as strong as that of the Earth, which would substantially reduce the evaporation rate by holding the ionized gas.

Finally we want to add a few words of caution. Although the most convincing interpretation of the low frequencies detected by Kepler in KIC 10001893 (and KIC 05807616) is the presence of Earth-size close-in planets, we do not consider these data and their analysis adequate to definitively prove that these extreme planetary systems around sdB stars do exist. Rather, we think that these data are enough to seriously pose the question. A final answer to this question will be given when it will be possible to confirm these detections with independent methods (e.g., RVs with PEPSI at LBT) and/or find further planetary systems similar to them. A program to search for planets/planetary remnants close to bright sdB stars has recently been started using Harps-N at TNG (Silvotti et al. 2014).

Acknowledgements. The authors gratefully acknowledge the Kepler team and everybody who has contributed to making this mission possible. Funding for the Kepler Mission is provided by NASA's Science Mission Directorate. R.S. was supported by the PRIN-INAF on "Asteroseismology: looking inside the stars with space- and ground-based observations" (PI Ennio Poretti). L.F.M. acknowledges the financial support from the UNAM under grant PAPIIT 104612. A.S.B. gratefully acknowledges a financial support from the Polish National Science Center under project UMO-2011/03/D/ST9/01914. V.V.G. is an FNRS Research Associate.

\section{References}

Baran, A. S., Kawaler, S. D., Reed, M. D., et al. 2011, MNRAS, 414, 2871 Baran, A. S., Reed, M. D., Stello, D., et al. 2012, MNRAS, 424, 2686 Bear, E., \& Soker, N. 2012, ApJ, 749, L14

Beuermann, K., Breitenstein, P., Bski, B. D., et al. 2012a, A\&A, 540, A8

Beuermann, K., Dreizler, S., Hessman, F. V., \& Deller, J. 2012b, A\&A, 543, A138

Borucki, W. J., Koch, D., Basri, G., et al. 2010, Science, 327, 977

Carter, J. A., Agol, E., Chaplin, W. J., et al. 2012, Science, 337, 556

Charpinet, S., Fontaine, G., Brassard, P., et al. 2011a, Nature, 480, 496

Charpinet, S., Van Grootel, V., Fontaine, G., et al. 2011b, A\&A, 530, A3
Fontaine, G., Brassard, P., Charpinet, S., et al. 2012, A\&A, 539, A12

Geier, S., \& Heber, U. 2012, A\&A, 543, A149

Geier, S., Schaffenroth, V., Drechsel, H., et al. 2011, ApJ, 731, L22

Geier, S., Classen, L., Brünner, P., et al. 2012, ASP Conf. Ser., 452, 153

Gilliland, R. L., Brown, T. M., Christensen-Dalsgaard, J., et al. 2010, PASP, 122, 131

Hambleton K. M., Kurtz D. W., Prsă A., et al. 2013, MNRAS, 434, 925

Han, Z., Podsiadlowski, Ph., Maxted, P. F. L., Marsh, T. R., \& Ivanova, N. 2002, MNRAS, 336, 449

Han, Z., Podsiadlowski, Ph., Maxted, P. F. L., \& Marsh, T. R. 2003, MNRAS, 341,669

Han, Z., Chen, X., Lei, Z., \& Podsiadlowski, P. 2012, PASP Conf. Ser., 452, 3

Hansen, C. J., Winget, D. E., \& Kawaler, S. D. 1985, ApJ, 297, 544

Heber, U. 2009, ARA\&A, 47, 211

Heber, U., Reid, I. N., \& Werner, K. 2000, A\&A, 363, 198

Kawaler, S. D., Reed, M. D., Quint, A., et al. 2010a, MNRAS, 409, 1487

Kawaler, S. D., Reed, M. D., Østensen R. H., et al. 2010b, MNRAS, 409, 1509

Lee, J. W., Kim, S.-L., Kim, C.-H., et al. 2009, AJ, 137, 3181

Lutz, R., 2011, Ph.D. Thesis, Georg-August-Universität Göttingen,

http://webdoc.sub.gwdg.de/diss/2011/lutz/lutz.pdf

Mustill, A. J., \& Villaver E. 2012, ApJ, 761, 121

Nelemans, G., \& Tauris, T. M. 1998, A\&A, 335, L85

Nordhaus, J., \& Spiegel, D. S. 2013, MNRAS, 432, 500

Nordhaus, J., Spiegel, D. S., Ibgui, L., Goodman, J., \& Burrows, A. 2010, MNRAS, 408, 631

Østensen R. H., Silvotti, R., Charpinet, S., et al. 2010, MNRAS, 409, 1470

Østensen, R. H., Silvotti, R., Charpinet S., et al. 2011, MNRAS, 414, 2860

Østensen, R. H., Degroote, P., Telting, J. H., et al. 2012, ApJ, 753, L17

Passy, J. C., Mac Low, M.-M., \& De Marco, O. 2012, ApJ, 759, L30

Qian, S.-B., Zhu, L.-Y., Zola, S., et al. 2009, ApJ, 695, L163

Qian, S.-B., Zhu, L.-Y., Dai, Z.-B., et al. 2012, ApJ, 745, L23

Reed, M. D., Kawaler S. D., Østensen R. H., et al. 2010, MNRAS, 409, 1496

Reed, M. D., Baran, A., Quint, A. C., et al. 2011, MNRAS, 414, 2885

Reed, M. D., Baran, A., Østensen R. H., Telting, J., \& O’Toole, S. J. 2012, MNRAS, 427, 1245

Schaffenroth, V., Geier, S., Heber, U., et al. 2014, A\&A, 564, A98

Schuh, S., Silvotti, R., Lutz, R., \& Kim, S.-L. 2014, ASP Conf. Ser., 481, 3

Shibahashi H., \& Kurtz, D. W. 2012, MNRAS, 422, 738

Silvotti, R., Schuh, S., Janulis, R., et al. 2007, Nature, 449, 189

Silvotti, R., Østensen, R. H., Telting, J. H.., \& Lovis, C. 2014, ASP Conf. Ser., 481,13

Soker, N. 1998, AJ, 116, 1308

Spiegel D. S. 2012, Proc. of conf. on Planets Around Stellar Remnants [arXiv: 1208.2276]

Telting J. H., Østensen R. H., Baran, A. S., et al. 2012, A\&A, 544, A1

Valencia, D., Ikoma, M., Guillot, T., \& Nettelmann, N. 2010, A\&A, 516, A20

Van Grootel, V., Charpinet, S., Fontaine, G., et al. 2010, ApJ, 718, L97

Villaver, E., \& Livio, M. 2007, ApJ, 661, 1192

Villaver, E., \& Livio, M. 2009, ApJ, 705, L81

Villaver, E., Livio, M., Mustill, A. J., \& Siess, L. 2014, ApJ, 794, 3 